\title{
Cognitive Recovery and its Correlation with Functional Status One Year After Traumatic Brain Injury
}

\author{
Mahima Agrawal, Mrinal Joshi \\ Department of Physical Medicine and Rehabilitation, S.M.S.M.C and hospital, Jaipur, Rajasthan, India
}

Email address:

docmahima@yahoo.com (M. Agrawal),dr_mrinal_joshi@hotmail.com (M. Joshi)

To cite this article:

Mahima Agrawal, Mrinal Joshi. Cognitive Recovery and its Correlation with Functional Status One Year After Traumatic Brain Injury. American Journal of Nursing Science. Vol. 4, No. 3, 2015, pp. 113-118. doi: 10.11648/j.ajns.20150403.20

\begin{abstract}
Objectives: To examine the impact of multi faceted rehabilitation services on functional outcomes after traumatic brain injury (TBI) for individuals with moderate and severe TBI. Design: Cohort, non-randomized, interventional study, pre-test, post-test design. Setting: Community integrated post acute rehabilitation centre. Main outcome measures: Galvenston Orientation and Amnesia Test (GOAT), Mini Mental Status Examination (MMSE), Functional Independence Measure (FIM), Glasgow outcome scale extended (GOSE). Results: Mean change in MMSE scores from admission to twelve months was 23.48 in moderate injury group and 21.26 in severe injury group. This change was significant in both the severity groups. Mean change in GOAT scores from admission to twelve months was 74.85 in moderate injury group and 70.71 in the severe injury group which also was significant. Predictors such as Length of stay (LOS) in acute care $(B=-0.913)$, GCS at the time of acute admission $(B=0.402)$, FIM at the time of discharge $(B=.241)$, injury severity $(B=0.221)$ and LOS in rehabilitation $(B=0.168)$ explained $85.7 \%$ of the variation in the value of FIM at 12 months after injury. Conclusion: Impairment of cognitive function and its socio-economic impact is vast such that it affects the socio economic status of a community and country as a whole. Cognitive rehabilitation remains central to the management of persons with TBI.
\end{abstract}

Keywords: FIM, GOSE, MMSE, GOAT, Cognitive, Rehabilitation, PTA (Post Traumatic Amnesia)

\section{Introduction}

Cognition is defined as the process of knowing through the selection and acquisition of that information, the understanding of that information and the application of knowledge in the appropriate situation. ${ }^{1}$ Cognitive rehabilitation therapy (CRT) is basically a broad term that covers a wide range of systematic, goal oriented approaches to overcoming or compensating for cognitive impairments such as those caused by TBI. ${ }^{2}$

TBI constitutes a major global health and socio-economic problem. At least 10 million traumatic brain injuries serious enough to result in death or hospitalization occur annually and an estimated 57 million people worldwide have experienced one or more TBI's. There is accumulating evidence that a subset of TBI's trigger progressive degenerative processes affecting cognitive or motor function or both. ${ }^{3}$

Impairments of cognitive function are amongst the most important issues that concern a TBI survivor. Considering the serious impact of cognitive deficits on safety, independence, social integration and financial status of a TBI survivor, comprehensive management of cognitive dysfunction forms an integral part of their treatment and recovery. Despite this, these individuals face difficulties in obtaining treatment for cognitive dysfunction termed cognitive rehabilitation.

We have authored this paper to address the need for medical and therapeutic needs of a TBI survivor for cognitive dysfunction, impact of cognitive rehabilitation on cognitive recovery and functional status one year after injury and research evidence for the efficacy of treatments.

\section{Methods and Materials}

\subsection{Evaluation of Study Subjects}

All individuals were subjected to standard neurological and functional examination in the acute care and rehabilitation setting. All were subjected to structured interviews, questionnaire and behavioral observations in our setting.

\subsection{Inpatient Rehabilitation}

All individuals received interdisciplinary rehabilitation administered by a specialized brain injury rehabilitation team 
comprising of a physiatrist, occupational therapist, and physical therapist, along with psychiatric consultations as per the requirement.

Our study group was filtered with regards to timing and etiology of brain injury, therefore, treatment was tailored according to individual differences in cognitive capacity and personality, academic and vocational assessment. All individuals were self-funded. Our focus was to optimize recovery and educate the patients and their families regarding the consequences of brain injury and the goals of management. In general, discharge from rehabilitation was decided when the patients reached a plateau of clinical improvement or achieved their treatment goals.

\subsection{Outpatient Rehabilitation}

Fifty eight patients could be followed up to twelve months post injury. After discharge, all patients were reviewed at an interval of every two months and appropriate medical and allied health treatments were given for aggressive behavior, emotional labiality, reduced attention and memory deficits. Custom physical therapy and orthotic measures were taken at appropriate times.

\subsection{Independent Variables}

The following baseline information was recorded in all patients: gender, age, sex, education (illiterate, $<5^{\text {th }}$ stnd, $>5^{\text {th }}<12^{\text {th }},>12^{\text {th }}$ stnd.), previous head injuries (yes/no), injury localization extracted from the clinical description of the head CT scan (frontal/non-frontal region), injury severity (severe/moderate), length of stay in acute hospital (LOS acute), length of stay in rehabilitation hospital (LOS rehab), PTA duration, Glasgow Coma Scale (GCS) at admission to acute hospitalization (GCS acute), and GCS at admission to the rehabilitation unit (GCS rehab).

\subsection{Inclusion Criteria}

Individuals with age greater than 16 years, injury severity classified as moderate and severe according to American college of rehabilitation medicine (ACRM) ${ }^{4}$ definition and those who gave consent for rehabilitation interventions were included in the study.

\subsection{Exclusion Criteria}

TBI classified as mild according to ACRM definition or associated serious co-morbidities such as associated spinal cord injuries, previously diagnosed severe psychological and neuropsychiatric disorders and/or substance abuse.

\subsection{Outcome Measures}

Each individual was subjected to neuropsychiatric assessment on MMSE and GOAT, once in one week from the date of admission in acute care facility to date of discharge from rehabilitation, and once in two months after discharge to one year post injury. Final outcome was measured on FIM and GOSE.
MMSE consists of 11 simple questions or tasks. Typically, these are grouped into 7 cognitive domains; orientation to time, orientation to place, registration of three words, attention and calculation, recall of 3 words, language and visual construction. Maximum score is 30 . A score of $23 / 24$ is the generally accepted cutoff point indicating the presence of cognitive impairment.5

PTA duration was defined as the interval in days between injury and obtaining a normal score on the GOAT. ${ }^{5}$ The GOAT 10-item scale, evaluates the major spheres of orientation (time, place, and person) and the ability of the patient to recall personal information and facts about recent events following injury. As such the GOAT assesses temporal orientation concerning the day of week, day of month, year, and time of day. A total score of $76-100$ indicates that a person is oriented, while a total score of 65 or less indicates impaired orientation. ${ }^{6}$

FIM was used to measure functional levels at admission and discharge from sub-acute rehabilitation as well as 12 months after injury. The FIM is an 18-item rating scale assessing self-care, bowel and bladder management, mobility, communication, cognition, and psychosocial adjustment. ${ }^{7}$

\subsection{Statistical Analysis}

The difference is calculated between values at: admission and discharge, admission and $1^{\text {st }}$ follow up, admission and $2^{\text {nd }}$ follow up and $1^{\text {st }}$ follow up and $2^{\text {nd }}$ follow up within each severity group. One-sample T-Test is applied taking all these 8 variables (separately for moderate and severe) as test variables. One way Anova is applied taking the above 4 data lists as dependent variable and Injury Severity as the grouping factor.

Recovery prediction is measured as the difference between the value of the factor at admission and on discharge. Correlation measured is Pearson Correlation. Regression was done with FIM at 12 months as the dependent variable and independent variables as shown in table IV. The adjusted $\mathrm{R}$ square for the regression is 0.857 , which shows that the independent variables together explain $85.7 \%$ of the variation in the value of FIM at 12 months. Also, the significance value is 0.000 , which implies that the regression model is statistically significant at $5 \%$ level of significance. FIM score at admission to sub-acute rehabilitation was included as a covariate to adjust for the baseline level.

Approval by ethical committee: This study was approved by ethics committee of Sawai Mansingh hospital, Jaipur on $4^{\text {th }}$ June 2011 with reference number 3681 and is in accordance with the Declaration of Helsinki.

\section{Results}

Seventy patients were included initially, 27 with moderate and 43 with severe injury. Of them 3 died in the acute care facility and 9 did not complete the follow up. The number of subjects for data analysis was thus reduced to 58 . 


\subsection{Demographics and Severity of TBI}

We had a total of 70 patients of which 64 were males and 6 were females. Of this $50(71.4 \%)$ were in the age group of (18-40) years, $15(21.43 \%)$ were in the age group of (41-60) years and 5 were above 60 years. Amongst the injured, 48 $(69 \%)$ were married and $44(63 \%)$ lived in joint families. Mode of trauma in $65(93 \%)$ was road traffic accidents (RTA) and in $5(7 \%)$ it was fall from height (FFH). Nearly $18(31 \%)$ had diffuse axonal injury (DAI) and rest $52(69 \%)$ had other forms of injury in which non frontal contusions was the most common in both the groups.

\subsection{Lengths of Inpatient Rehabilitation}

Transfer to the rehabilitation unit took place on an average of 6.7 days after trauma in moderate group and 11.74 days in severe injury group. Average length of stay in rehabilitation unit in moderate group was 13.37 days and in severe group 28.8 days.

\subsection{Measurements of Cognitive Recovery and Functional Status}

In moderate injury group, MMSE and GOAT were testable in 19 individuals at the time of rehabilitation admission as against 8 in the severe group. Average days after which both the scales became testable were 7.30 and 20.97 in moderate and severe group respectively. Mean MMSE scores were $19.89 \pm 7.07$ in moderate group and $14.53 \pm 7.73$ in the severe group at the time of discharge. All in moderate injury group achieved scores greater than 24 while 6 in severe injury group still had scores less than 24 at 12 months and 1 did not improve at all. The mean change in MMSE scores at different time frames is shown in table I.

Table I. Comparison of Moderate and Severe group with respect to change in MMSE score in different time frames.

\begin{tabular}{|c|c|c|c|c|c|c|}
\hline \multicolumn{7}{|c|}{ Mini Mental Status Examination (MMSE) } \\
\hline \multirow{2}{*}{ Type of Injury } & \multicolumn{2}{|c|}{ Admission to Discharge } & \multicolumn{2}{|c|}{ Admission to Ist Follow-up } & \multicolumn{2}{|c|}{ Admission to IInd Follow-up } \\
\hline & Mean Change & P Value & Mean Change & P Value & Mean Change & P Value \\
\hline Severe & 10.32 & 0.000 & 19.32 & 0.000 & 21.26 & 0.000 \\
\hline Moderate-Severe & - & 0.014 & - & 0.042 & - & 0.184 \\
\hline
\end{tabular}

Mean GOAT scores were $62.22 \pm 22.74$ in moderate group and $38 \pm 28.26$ in severe injury group at the time of discharge. Despite these low scores at the time of discharge in severe group, there was no significant difference in both the groups at 12 months on both the scales. Mean change in GOAT scores at different time frames is shown in table II.

Table II. Comparison of Moderate and Severe group with respect to change in GOAT score in different time frames.

\begin{tabular}{lllllll}
\hline \multicolumn{2}{l}{ Galvenston Orientation and Amnesia Test (GOAT) } & & & \\
\hline \multirow{2}{*}{ Type of Injury } & \multicolumn{2}{l}{ Admission to Discharge } & \multicolumn{2}{l}{ Admission to Ist Follow-up } & \multicolumn{2}{l}{ Admission to IInd Follow-up } \\
\cline { 2 - 7 } & Mean Change & P Value & Mean Change & P Value & Mean Change & P Value \\
\hline Moderate & 52.44 & 0.000 & 72.07 & 0.000 & 74.85 & 0.000 \\
Severe & 32.32 & 0.000 & 64.03 & 0.000 & 70.71 & 0.000 \\
Moderate-Severe & - & 0.005 & - & 0.119 & - & 0.184 \\
\hline
\end{tabular}

The FIM scores of all individuals at 12 months follow up, in moderate and severe group were greater than 106 i.e. all were independent in their daily activities except one in severe group who did not improve at all. But the total mean scores in severe group (118.6 $5 \pm 19.11)$ at 12 months were lower than moderate group $(124.26 \pm 3.97)$. The recovery patterns on FIM are shown in table III.

Table III. Comparison of Moderate and Severe group with respect to change in FIM score in different time frames.

\begin{tabular}{lllllll}
\hline \multicolumn{2}{l}{ Functional Independence Measure (FIM) } \\
\hline \multirow{2}{*}{ Type of Injury } & \multicolumn{2}{l}{ Admission to Discharge } & \multicolumn{2}{l}{ Admission to Ist Follow-up } & \multicolumn{2}{c}{ Admission to IInd Follow-up } \\
\cline { 2 - 7 } & Mean Change & P Value & Mean Change & P Value & Mean Change & P Value \\
\hline Moderate & 51.26 & $<0.0001$ & 84.78 & $<0.0001$ & 85.3 & 0.0104 \\
Severe & 54.52 & $<0.0001$ & 93.96 & $<0.0001$ & 97 & 0.0104 \\
Moderate-Severe & - & 0.627 & - & 0.139 & - & 0.060 \\
\hline
\end{tabular}

Assessment on GOSE revealed that in moderate injury group, only 3 had moderate disability at 12 months post injury, rest all had good recovery. In the severe injury group, at the end of 12 months, 16 had good recovery and 14 had moderate disability while 1 still had severe disability.

On regression analysis of independent variables, the p-value is less than 0.05 for GCS Scores, LOS Acute, LOS
Rehabilitation, Injury Severity and FIM at discharge. This means that of all the independent variables considered; only these 5 variables are significant in predicting the recovery pattern at the time of admission as measured by the FIM at 12 months, as seen in table IV. These 5 values account for $85.7 \%$ of the variation in the value of FIM at 12 months. 
Table IV. Independent variables on regression analysis.

\begin{tabular}{lll}
\hline Variable & B Coefficient $\mathbf{( 9 5 \% ~ C I ) ~}$ & P-value \\
\hline Constant & 134.442 & .000 \\
GCS score & +0.402 & .000 \\
PTA duration & -0.012 & .895 \\
Age group & -0.103 & .083 \\
Sex & 0.286 & .608 \\
LOS acute & -0.913 & .000 \\
LOS rehab & +0.168 & .012 \\
Education & +0.016 & .775 \\
Injury severity & +0.221 & .021 \\
FIM at admission & +0.066 & .321 \\
FIM at discharge & +0.241 & .001 \\
\hline
\end{tabular}

\section{Discussion}

Initially after brain injury, arousal, attention and registration may be the most obvious issues; later social involvement and executive functioning may become prominent. Considering the different pace of recovery for each individual, some people return to premorbid levels of functioning whereas others may have permanent cognitive problems. Cognitive rehabilitation is a systematically applied set of medical and therapeutic services designed to improve cognitive functioning and participation in activities that may be affected by difficulties in one or more cognitive domains. 8

Wellmark blue cross blue shield of Iowa concludes that cognitive rehabilitation may be performed by a physician, psychologist or a physical, occupational or speech therapist. 9

Denial of cognitive rehabilitation in neurosurgery and trauma is usually attributed to the paucity of definitive evidence for efficacy. But lately, the vastness of literature on cognitive rehabilitation has grown and now incorporates more than 770 studies supporting the efficacy of the same. The National institutes of health $(\mathrm{NIH})$ convened a consensus development conference in 1998 to report on the scientific basis of therapeutic interventions for sequel of brain injury. 10 Carney and colleagues as part of NIH consensus development conference found limited evidence to support certain forms of cognitive rehabilitation in treating memory and anxiety and in improving self concept and interpersonal relationships. They cautioned that long term benefit and clinical relevance were not well established. 11

In this study we have documented the time course and magnitude of cognitive recovery during the first six months and first one year and functional outcome after one year of TBI in a group of 70 patients. There were twelve drop outs, therefore 58 patients with moderate $(n=27)$ and severe $(n=$ 31) TBI, were included in the study.

Cognitive status as assessed on MMSE improved significantly during first twelve months with maximum improvement occurring during inpatient rehabilitation phase. This was on an average 45 days for severe TBI and 30 days for moderate TBI in our setting, and this is in close agreement with the literature. Though recovery on this ground continued up to 12 months and was significant in all time frames but this occurred at a slower pace after discharge. Previous studies have documented that cognitive recovery after TBI is most rapid in the first 5 months after injury and continues at a slower rate in the next 7 months. 12

The improvement in cognitive status in our study was significant even from 6 to 12 months. These results suggest the efficacy of the intensive rehabilitation that was offered to these patients even in the outpatient setting. This is not in agreement with other studies as that done by Maria Sandhaug and colleagues. 13 The lack of improvement in the later phase found in their study could be due to the fact that FIM is not sensitive enough to detect changes in either upper functional level or light cognitive dysfunction. 14

Difference in between moderate and severe group was significant from admission to discharge and 6 months but not after that. This is because of the slower recovery in patients with severe TBI as against those with moderate injury. The final scores in severe group were lower at all times in severe injury.

Though, MMSE has been used widely as a tool to measure cognitive impairment but Shrivastava et al suggest that MMSE should not be used to identify those with cognitive impairment. If administered, a perfect score on a component of MMSE suggests that enhanced testing in that given domain may not reveal further impairment. 15

Similar results were obtained on GOAT assessment. Significant improvements were seen at all time frames, maximum being in the inpatient rehabilitation phase. The difference in scores between moderate and severe group were significant from admission to discharge and from 6 months to 12 months as against the finding on MMSE. This is due to differences in sensitivity of the two scales in assessing mental status. 16,17

The mean change in FIM scores from discharge to 6 months was significant in both the groups but post six months it improved significantly only in severely injured group as against the moderately injured in which no significant change occurred. This finding can be explained by the lower status in severe group at the time of admission and discharge, though the final scores were lower at all times in severely injured group. This difference between moderate and severe group was not significant at any time during the course of one year on unpaired $\mathrm{T}$ test ( $\mathrm{P}$ value at 12 months $=0.060$ ). GOSE is often considered to be a better assessment of long term functional outcome after TBI than FIM. 18

Correlation between different scales as seen on Pearson correlation analysis showed that MMSE and GOAT are strongly positively correlated, $(\mathrm{r}=0.778)$. FIM and GOSE are slightly positively correlated $(\mathrm{r}=0.298)$. Relation of MMSE and GOAT with FIM and GOSE was not significant in our study which is against the finding of JTL Wilson and colleagues according to which there is a modest correlation between cognition and functional status. 19

The significant improvement seen in our study during the inpatient rehabilitation phase and thereafter contribute some evidence to the efficacy of cognitive rehabilitation in particular and comprehensive rehabilitation in general. This statement is supported by a number of studies. Cicerone KD et al published an evidence based review of the cognitive 
rehabilitation literature in 2000 and a comprehensive updated review in 2005, that included treatments studied in TBI and stroke survivors. They concluded that there is substantial evidence to support cognitive rehabilitation for people with brain injury. 20, 21

Gordon et al further reviewed 28 studies and provided further evidence for the efficacy of cognitive rehabilitation. 22 Similarly, positive conclusions were drawn in a recent volume devoted to the effectiveness of cognitive rehabilitation that was based on an international conference commenced in 2002. 23

LOS Acute in our regression analysis was found to be the strongest predictor $(B=-0.913)$ of functional recovery at 12 months as compared to LOS rehabilitation which was found to be a weak predictor $(B=-0.168)$. A study by Cowen and colleagues stated that longer acute hospitalization LOS resulted in significantly lower FIM motor and cognitive scores on admission to rehabilitation. Using the same analysis, longer acute hospitalization was strongly associated with longer rehabilitation LOS. 24

The GCS is the most widely used clinical assessment of TBI severity. $25 \mathrm{It}$ is also used in predicting late global outcome such as functional level and return to employment. 26 In our study, GCS within 72 hours of acute care admission was taken as a long term functional predictor. On regression analysis, GCS Scores were the second strongest predictor $(B=0.402)$ of functional level at 12 months after injury, as against the study of Maria Sandhaug and colleagues. 18 This discrepancy could be due to the difference in time periods in which GCS was recorded.

PTA is the interval from injury to return of orientation or continuous memory. 27 In our model, PTA was found to be a weak predictor $(-0.103)$ of functional recovery which is in close agreement with the study done by Maria Sandhaug and colleagues. 18 This finding is against that documented in the literature. 28,29

The growing evidence of literature on cognitive rehabilitation whether positive or negative emphasizes the need to strengthen the evidence and answer specific questions regarding the method of rehabilitation. Changes in brain organization do not occur immediately but are subject to long term interaction and require minimal period of training. 30

There are clear implications of these findings for the role of rehabilitation in cognitive and motor recovery after brain injury. Laastch and colleagues recently demonstrated cortical reorganization following cognitive rehabilitation in five patients who showed marked enhancement in fMRI activity in brain areas related to the tasks being trained. 31

The current paper extends these recommendations by suggesting that incorporation of techniques such as Coma stimulation program, memory boosting techniques, behavioral counseling and management of attention deficits are possibly effective. Research in this area is made difficult by numerous factors including the ability to isolate and define the therapeutic ingredients of these therapeutic interventions and the need to use of a variety of relevant health related outcome measures reflecting participant's social participation and well being.

Though the major issues in TBI rehabilitation were addressed in our study but we could not analyze behavioral deficits on standard scales due to technical fallacies. Also, our sample size cannot be used to draw generalized conclusions. Having controls in such a study was not possible on ethical grounds. The strength of our study was large and complete follow up and lesser drop outs.

\section{Conclusions}

Impairments of cognitive function and their socio economic impact is vast such that it affects the entire economy of the country. Though there are gaps in our knowledge for effectiveness of cognitive rehabilitation but these research questions should not hamper us from providing such treatment. Cognitive rehabilitation remains central to the management of persons with acquired brain injury as has been well proven by our study. All individuals improved significantly during the time frame from admission to discharge from the inpatient rehabilitation facility. The residual cognitive and overall impairment seen in the individuals post discharge emphasize the importance of follow up. Thus, this process should be integrated into and coordinated with vocational services and community based programming so that individuals move effortlessly within a comprehensive and a coordinated system of care that is adequately funded.

\section{Acknowledgements}

We would like to thank Prof. Dr. S.K. Jain at department of neurosurgery, SMS hospital Jaipur his support with patient referral and statistician Miss. Ruby Pasricha, for her supervision in statistics.

\section{References}

[1] G. Eason, B. Noble, and I. N. Sneddon, "On certain integrals of Cognitive rehabilitation for traumatic brain injury and stroke, updated review of literature from 1998 through 2002 with recommendation for clinical practice.

[2] Cognitive rehabilitation therapy may assist in treating traumatic brain injury. Medical news today. 13 Oct 2011. 10.00 PDT

[3] Institute of medicine. Gulf war and health: Volume 7: long term consequences of TBI. Washington (DC): National academy Pr; 2009

[4] Ruff RM, Iverson GL, Barth JT, Bush SS, Broshek DK. Recommendations for diagnosing a mild traumatic brain injury: a National Academy of Neuropsychology education paper. Arch Clin Neuropsychol 2009; 24(1):3-10

[5] Dick JP, Guiloff RJ, Stewart A, Blackstock J, Bielawska C, Paul EA, Marsden CD. Mini-mental state examination in neurological patients. J Neurol Neurosurg Psychiatry. 1984 May;47(5):496-9 
[6] Levin HS, O'Donnell VM, Grossman RG. The Galveston Orientation and Amnesia Test. A practical scale to assess cognition after head injury. J Nerv Ment Dis. 1979 Nov;167(11):675-84

[7] Grimby G, Gudjonsson G, Rodhe M, Sunnerhagen KS, Sundh $\mathrm{V}$, Ostensson ML. The functional independence measure in Sweden: experience for outcome measurement in rehabilitation medicine. Scand J Rehabil Med. 1996 May;28(2):51-62

[8] Katz, DI; Ashley MJ, O'Shanick GJ, Connors, SH. Cognitive rehabilitation: the evidence, funding and case for advocacy in brain injury. McLean, VA: Brain Injury Association of America, 2006

[9] WWW.Wellmark.com/ e business/ provider/ medical policies/ cognitive rehab. htm

[10] Bethesda MD. National institute of health. Report of the consensus development conference on rehabilitation of persons with traumatic brain injury. Sep. 2009

[11] Carney N, Chestnut RM, Maynard H, mann NC, Patterson P, Helfand M. Effect of cognitive rehabilitation on outcomes for persons with traumatic brain injury: A systematic review. J Head Trauma Rehabil. 1999 Jun;14(3):277-307

[12] Christensen BK, Collela B, Inness E, Herbert D. Recovery of cognitive function after traumatic brain injury: a multilevel modeling analysis of Canadian outcomes. Arch Phys Med Rehabil. 2008. Dec; 89(12 suppl):S3-15.

[13] Bilbao A, Kennedy C, Chatterji Set al. The ICF: Applications of the WHO model of functioning, disability and health to brain injury rehabilitation. Neuro Rehabilitation 2003.18(3):239-250.

[14] Kunik CL, Flowers L, Kazanjian T. Time to rehabilitation admission and associated outcomes for patients with traumatic brain injury. Arch Phys Med Rehab 2006.Dec;87(12):1590-1596

[15] Srivastava A, Rapoport MJ, Leach L, Phillips A, Shammi P, Feinstein A. The utility of the mini-mental status exam in older adults with traumatic brain injury. Brain Inj. 2006 Dec;20(13-14):1377-82

[16] Katz DI, Alexander MP. Traumatic brain injury. Predicting course of recovery and outcome for patients admitted to rehabilitation. Arch Neurol. 1994 Jul;51(7):661-70

[17] Tombaugh TN, McIntyre NJ. The mini-mental state examination: a comprehensive review. J Am Geriatr Soc. 1992 Sep;40(9):922-35

[18] Sandhaug M Andelic N, Bernsten SA, Seiler S, Mygland A. Functional level during the first year after moderate and severe Traumatic Brain Injury: Course and Predictors of Outcome. J Neurol Res 2011.1(2): 48-58
[19] J T L Wilson, L E L Pettigrew, G M Teasdale. Emotional and cognitive consequences of head injury in relation to the Glasgow outcome scale. J Neurol Neurosurg Psychiatry 2000 Aug;69:(2);204-209

[20] Cicerone KD, Dahlberg C, Kalmar K et al. Evidence based cognitive rehabilitation: recommendations for clinical practice. Arch Phys med Rehabil. 2000 Dec;81(12):1596-615

[21] Cicerone KD, Dahlberg C, Malec JF et al. Evidence based cognitive rehabilitation: updated review of literature from 1998 through 2002. Arch Phys med Rehabil. 2005 Aug;86(8):1681-92.

[22] Gordon WA, Zafonite R, Cicerone K, Cantor J, Brown M, Lombard L, Goldsmith R, Chandan T. Traumatic brain injury rehabilitation: state of the science. Amer J Phys Med Rehabil. 2006; 85(4): 345-82

[23] Halligen PW, wade DT, (Eds.). Effectiveness of rehabilitation for cognitive deficits. Oxford university press 2005

[24] Cowen TD, Meythaler JM, DeVivo MJ, Ivie CS 3rd, Lebow J, Novack TA. Influence of early variables in traumatic brain injury on functional independence measure scores and rehabilitation length of stay and charges. Arch Phys Med Rehabil. 1995 Sep;76(9):797-803

[25] The Brain Trauma Foundation. The American Association of Neurological Surgeons. The Joint Section on Neurotrauma and Critical Care. Glasgow coma scale score. J Neurotrauma 2000;17(6-7):563-571

[26] Dikmen SS, Ross BL, Machamer JE, Temkin NR. One year psychosocial outcome in head injury. J Int Neuropsychol Soc 1995;1(1):67-77

[27] Sherer M, Struchen MA, Yablon SA, Wang Y, Nick TG. Comparison of indices of traumatic brain injury severity: Glasgow Coma Scale, length of coma and post-traumatic amnesia. J Neurol Neurosurg Psychiatry 2008;79(6):678-685

[28] Ariza M, Mataro M, Poca MA, Junque C, Garnacho A, Amoros $\mathrm{S}$, Sahuquillo J. Influence of extraneurological insults on ventricular enlargement and neuropsychological functioning after moderate and severe traumatic brain injury. J Neurotrauma 2004;21(7):864-876

[29] Katz DI, Alexander MP. Traumatic brain injury. Predicting course of recovery and outcome for patients admitted to rehabilitation. Arch Neurol 1994;51(7):661-670

[30] Kleim JA, Hogg TM, VandenBerg PM, Cooper NR, Bruneau R, Remple M. Cortical synaptogenesis and motor map reorganization occur during late, but not early, phase of motor skill learning. J Neurosci. 2004 Jan 21;24(3):628-33

[31] Laatsch LK, Thulborn KR, Krisky CM, Shobat DM, Sweeney JA. Investigating the neurobiological basis of cognitive rehabilitation therapy with fMRI. Brain Inj. 2004 Oct;18(10):957-74 